\title{
Supporting 21st-Century Teaching and Learning: The Role of Google Apps for Education (GAFE)
}

\author{
Lawrence J. Awuah
}

The future of higher education is likely to be driven by to the willingness to adapt and grow with the use of technologies in teaching, learning, and research. Google Apps for Education (GAFE) is a powerful cloud-computing solution that works for students regardless of their location, time, or the type of device being used. GAFE is used by thousands of schools and universities worldwide to make effective use of collaboration tools for students and faculties, with the primary objective of enhancing teaching and learning. In particular, GAFE tools enable users to work together virtually on documents, presentations, and projects in the cloud. GAFE is used to develop course websites, as a complement to traditional classroom instruction, with the aim of delivering coursework to students.

For this research study, a group of computer science students from the University of Ghana were surveyed to understand the impact of GAFE use on their performance and satisfaction. The study was conducted after in-class deployment of GAFE during the fall 2013 semester. When asked if using the GAFE-based course website improved their performance, over 84\% answered "yes." Additionally, about 91\% of the participants indicated they were more satisfied with the courses using GAFE than those using traditional methods of instruction with no or moderate use of technology, where the least proportion of content is delivered online; the remaining 9\% noted they were moderately satisfied. Overall, the respondents were satisfied to some degree.

The success of higher education is driven by the ability to adapt, change, and grow with technology use. Colleges and universities undergo significant changes to acclimate to and utilize current innovations. This non-experimental study was conducted to determine if using GAFE for pedagogically-related objectives could impact student performance and adoption behaviors. Specifically, the researcher investigated whether using the course website, along with Google suites of apps, enhanced teaching, research, and learning on campus.

A group of students from the University of Ghana (UG) were selected as research participants after GAFE use during one semester. In addition, the impact on student performance as a result of using this innovation was investigated. In other words, the study explored the role that Google Apps plays in 21st-century education. The critical factors leading to users' adoption of innovations have inspired several streams of research over the decades. According to Allen and Seaman (2014), most academic institutions are currently securing efficient ways of employing web-based instruction systems for designing and delivering their courses online as a result of the rapid growth of educational apps and e-learning platforms. According to Sloan Consortium (2013), online courses constitute a model of instruction where at least 80 percent of course content is delivered online.

Recognizing the potential of educational apps, 
many academic institutions are embracing a variety of apps as an innovative way of strengthening collaboration among students and academic staff. These apps are being used either to engage students in traditional classroom instructions or as a supplement to online learning, as they have been at the forefront of supporting global knowledge building.

With the rapid development and accessibility of educational apps, there has been a significant rise in the adoption of these apps by educators, but the level of use could be higher. A range of emerging apps are published on the market every day, and, according to Enis (2013), as the popularity and functionality of apps continue to grow, there is increased collaboration among students and faculty. For example, wikis have been noted as a social factor in facilitating effective collaboration $(\mathrm{Fu}, \mathrm{Chu}$, \& Kang, 2013).

These apps are fundamentally interactive computer programs designed to achieve particular usability needs with inherent advantages. Apps (short for applications) can be defined as a set of interrelated software programs, a self-contained program, or simply an interactive computer program designed to process data to accomplish a particular purpose that accounts for specific usability goals of the user. The usability goals, such as perceived ease of use, perceived usefulness, perceived effectiveness, as well as perception about safety and utility of the app are key determinants of the user's intention to use and adopt a specific app or technology in general. When an app is designed for pedagogical use, it tends to bridge the gap between education and technology with the exclusive drive to advance teaching, research, learning, and administration of schools. From this definition, GAFE can thus be described as an integrated suite of cloud-based solutions, driven by Google app engines, designed to achieve specific educational goals with the aim of transforming the 21st-century educational system.

Google Apps were designed to facilitate the provisioning of the Google suite of applications and other collaborative tools, such as Gmail, Google Drive, Google Sites, Google Calendar, Google Docs, Google+, and Google Chat, among others.

To meet the challenge of 21st-century educational goals, dramatic change should be directed toward the applicability of apps in teaching, research, learning, and administration of colleges. Educational apps have been instrumental in trans- forming educational institutions. In mobile computing, for example, Android and iOS smartphone operating systems have made a tremendous impact and continue to change teaching and learning in higher education (Enis, 2013; Kaganer, Giordano, Brion, \& Tortoriello, 2013). Currently, a spectrum of competing apps are on the market, including mobile-specific apps such as Android apps, iOS apps, Google apps, Chrome apps, Chromecast apps, Microsoft's OneDrive apps, book apps (e.g., Kindle, CourseSmart, VitalSource), and other productivity apps. For example, book apps are designed to offer interactive content while allowing navigation with taps, finger swipes, and finger zooms on smartphones and tablets not feasible on a typical laptop or desktop (Scheuer, 2013). Over the years, there has been increased use of apps in the forms of blogs, wikis, shared documents, shared drives, RSS readers, and video sharing in the cloud with the aim of transforming teaching and learning $(\mathrm{Fu}$, Chu, \& Kang, 2013; Norman, Din, Nordin, \& Ryberg, 2014).

Additionally, these pedagogical apps play a key role especially when integrated into online learning platforms. According to the Babson Survey Research Group (as cited in Allen \& Seaman, 2014), the number of students taking at least one online course increased by over 411,000 to a total of 7.1 million from 2012 to 2013. The report further stated that the percentage of higher education students taking at least one online course was estimated at $33.5 \%$. Education has begun to adapt to the everchanging trend of emerging technologies through distance learning and online platforms (Hodge \& Harman, 2013), as well as the use of educational apps in higher education. There is increased pressure to provide academically diverse and competent students to improve performance with the delivery of science, technology, engineering, and mathematics (Israel, Marino, Basham, \& Spivak, 2013).

Besides performance, some online platforms have been designed to provide free and flexible access to learning, for example, massive open online course (MOOCs) with the primary objective of making education available to potential learners regardless of geographical, physical, and time boundaries (Ravi Shankar, 2012). According to Coursera (2014), this innovation enables universities to teach millions of students across the world 
regardless of their geographic location.

Social networking-related apps have been shown to impact teaching and learning as well. Gewerc, Montero, and Lama (2014) conducted a study on the impact of collaboration and social networking in higher education and concluded that social networking offers sufficient opportunities for collaboration and visibility. When social networking tools are used effectively, instructors, learners, and classrooms are transformed into a rich, interactive environment (Huffman, 2013). Additionally, Junco, Heiberger, and Loken (2011) and Veletsianos (2012) investigated the impact of Twitter on college student engagement and grades and found evidence to suggest that Twitter can engage students and faculty in the learning process through communication and connections. These apps provide benefits to users by being open-source and customizable with multitasking capabilities. In another study, Norman, Din, Nordin, and Ryberg (2014) found that mobile blogs could be used as a learning tool to enhance learning and instruction in higher education. CNET News reported that Microsoft offers the first seven gigabytes (GB) of storage to its users, the ability for users to receive up to five GB of free storage for referrals, three GB of storage to camera backup service users, and additional bonuses (as cited in Cooper, 2014).

Further, social networking platforms can be integrated into learning management systems, such as Blackboard, eCollege, LoudCloud, and Angel. These online learning applications are powerful, integrated platforms that help educators develop web-based programs to deliver state-of-the-art online courses. These platforms have been known to facilitate learning, communication, and collaboration (Despotović-Zrakić, Marković, Bogdanović, Barać, \& Krčo, 2012). Integration of Blackboard and Skype-based electronic mentoring systems and adapting learning management systems have been found to be beneficial to teachers (Suk Hwang \& Vrongistinos, 2012; Ucol-Ganiron, 2013).

In another study, Skype-based virtual coaching was found to develop teachers' use of evidencebased classroom management practices (Rock et al., 2013). Web 2.0-based social network services have been found to aid the development of informal learning, learning networks, video sharing, question-embedded interactive video environment tools, and online event scheduling (Pritchett, Woh- leb, \& Pritchett, 2013; Vural, 2013; Zolotukhin, 2013). Furthermore, LEARN365 fosters social learning by providing a virtual space for students and teachers to come together and exchange information (DiScipio, 2013).

While each of these competing apps brings accompanying advantages and disadvantages, the researcher believes that GAFE cut across these competing apps with the applicability of real-time, collaborative, and education-driven solutions to meet 21st-century educational goals. Google Apps have been central in facilitating collaboration and advancing knowledge. In addition, Google Docs facilitates ease of collaboration with multiple editors so users can simultaneously make changes to the same document in real time. Regarding time management and scheduling of collaborations, users now have the ability to add calendar entries directly from their Gmail accounts. Furthermore, Google Sites provides faculties and students both communication and collaboration capabilities to achieve optimum productivity within the classroom environment-both traditional brick-andmortar and virtual learning environments. In particular, the real-time editing in Google Groups can make it easier for students to work collaboratively to share research findings and work together on group projects regardless of their physical location. Consequently, GAFE was chosen for analysis in this study.

\section{UNDERSTANDING GOOGLE APPS FOR EDUCATION}

GAFE is a powerful cloud-computing solution that works no matter where students are or what devices are used (Google, 2013). The platform is used by thousands of schools and universities worldwide to make effective use of available collaboration tools for students and faculties with the primary objective of enhancing teaching and learning. Specifically, GAFE tools enable users to work together virtually on documents, presentations, and projects in the cloud. GAFE is used to develop course websites, as a complement to traditional class instruction, to efficiently deliver coursework to students. The potential of GAFE-based cloud computing for improving efficiency and reducing cost for pedagogical gain has been recognized by a number of U.S. educational establishments.

The GAFE suite comprises Gmail, Google Drive, Google Groups, Google Calendar, Google 
Docs, Google Sites, and Google+. Various editions of the Google Apps suite have been developed with specific targets such as government, business, and education (Google, 2013). Cost, reliability, storage, and the scope of services offered by GAFE are the primary considerations for adoption by most academic institutions ("Google Plans Google Play for Education,” 2013).

\section{TECHNOLOGY ACCEPTANCE AND GAFE}

Understanding faculties' and students' adoption of an innovation such as GAFE is an important topic, as the use of these apps has become an integral part of teaching and learning. The realization of such innovations generally depends on the effective use of these educational apps. Technology use has associated benefits, opportunities, and challenges for students, faculties, and school administrators within the academic domain. GAFE adoption and use continue to be a concern due to prevailing factors such as security, risk, trust, computer literacy, culture, digital divide, bandwidth, and access to the Internet. Recent research studies have been conducted on technology acceptance and use (e.g., Lee \& Song, 2013; Nistor, Göğüş, \& Lerche, 2013; Nistor, Lerche, Weinberger, Ceobanu, \& Heymann, 2014; Venkatesh, Thong, \& Xu, 2012). For example, trust and perceived risk were found to be direct determinants of intention to use a technological tool (Lee \& Song, 2013). Additionally, Venkatesh, Thong, and $\mathrm{Xu}$ (2012) confirmed the roles of hedonic motivation, price, and habit in impacting technology use, which is tailored to the context of consumer adoption and use of technology.

Moreover, increased internationalization of education where learners from different cultural backgrounds are involved in the learning process has resulted in different expectations based on design of technology-enhanced learning (Nistor et al., 2013; Nistor et al., 2014). In addition, Teo and Noyes (2014) found that the strength and influence of the determinants, such as performance expectancy and effort expectancy, on the behavioral intention to use technology may interact differently with respect to different technologies, user populations, and cultures. Evidence has suggested that individual differences based on age, gender, and computer experience moderate the effects of hedonic motivation, price, and habit on behavioral intention and technology use (Venkatesh et al., 2012). According to Lin,
Zimmer, and Lee (2013), adopting computer technology to enhance effective delivery of scholastic content required changes in the curriculum, teaching practices, and allocation of relevant resources. These technology acceptance studies conducted with different technologies in different contexts are applicable to the adoption and use of GAFE as educational tools.

\section{GAFE ADOPTION BY VARIOUS UNIVERSITIES}

A number of U.S. colleges and universities have partnered with Google to offer the GAFE platform to their students and faculties in an attempt to meet the challenges and opportunities of 21st-century teaching and learning. It is the objective of every academic institution to provide constructive solutions and viable options. For example, Arizona State University is determined to approach higher education using new methods of thinking and teaching with the adoption of emerging technologies, adeptly applied to accelerate the advancement of teaching and research (Barlow \& Lane, 2007). Furthermore, the customized versions of Google Docs, Calendar, and Spreadsheets allow the UG community to create and share documents and spreadsheets with one another, thus enhancing collaboration among students and instructors. As new applications for GAFE evolve, UG will continue to leverage these functionalities to improve the online experience and to enhance teaching and learning on campus.

Other universities have successfully made this transition. For example, Valparaiso University transitioned to the Google Apps platform from the GroupWise e-mail and calendar system (Klein, Orelup, \& Smith, 2012). The transition made an impact on communication and collaboration. Further, some school districts have used GAFE as collaborative tools for creating, sharing, and editing documents and scheduling (Robertson, 2013). With this cloudbased environment, teachers can receive support for increased productivity. According to Stein, Ware, Laboy, and Schaffer (2013), the cloud is capable of delivering services in a cost-effective manner while protecting access to these educational apps. Moreover, compared to traditional software development, Software as a Service allows organizations to subscribe to some applications from cloud providers without having to build and maintain the software themselves (Wang \& Jin, 2010). These institutions have been able to transition from their legacy 
server systems to Gmail, with the expectation of providing students more storage space as well as enhanced spam filtering, a calendar system, instant messaging, in addition to the ability to sort, search, and tag e-mails. Adoption of GAFE by some students, faculties, and college administrators can be challenging. Evidence has suggested that despite pedagogical and economic benefits enjoyed from cloud computing, any innovation can fail if its users are hesitant to adapt to changes (Stein et al., 2013). However, whether those users-faculties, general staff, and students-adopt these innovations remains a concern, as there are benefits to both students and faculty.

\section{BENEFITS OF GAFE}

GAFE has advantages that are perceived as transformational in the educational system. The adoption of online collaboration tools allows groups with common interests to share content via "wikis, discussion forums, and through various file formats that can be shared or edited online" (Cheung \& Vogel, 2013, p. 160). A typical example is how Google Docs facilitates ease of collaboration with multiple editors to simultaneously make changes to the same document in real time. Additionally, geographic location, platform dependency, and compatibility issues are no longer restraining factors. With revision history, users can easily see all changes with different time-stamps, compare different versions of the same document, merge documents, as well as revert to previous versions of a document. Additionally, users can use the chat tool for discussions, while the owner of the document can grant access rights to users with different roles such as viewers or collaborators. GAFE tools are powerful and easy to use and help "administrators manage things like users, documents and services, and keep track of usage and data via dashboards" (Google, 2013).

Considering time management and scheduling of collaborations, users now possess the ability to add calendar entries directly from their Gmail accounts. In addition, with Google+ (instant messaging, voice, or video calls, etc.), users receive automatic updates of Gmail contacts as a result of e-mail communications. The integrated GAFE tools have been used to resolve communication issues among students and academic staff (Sviridova, Sviridova, \& Tymoshenko, 2011) and maintain high productivity gains in the classroom.

Furthermore, Google Sites offers faculties and students both communication and collaboration capabilities to achieve optimum productivity within the classroom environment, both traditional brickand-mortar and virtual learning environments. For example, for this study, course websites were developed on Google Sites and pilot tested for a group of UG computer science students. When asked how satisfied they were with the courses with the GAFE-based course website, nearly 38\% of the respondents were extremely satisfied, while $53 \%$ were very satisfied. The remaining $10 \%$ of respondents noted that they were moderately satisfied. Overall, all respondents were satisfied to some degree. Through the integration of the GAFE-based course websites, Google Drive, Google Calendar, and Gmail, the participants stayed up-to-date about the course assignments and activities.

Another key benefit of GAFE is the Google Groups platform, which stimulates increased collaboration, threaded discussions, and Q\&A forums. In addition, Groups can be used to schedule meetings and project events among a group of classroom students, and students can read group posts through e-mail, the online interface, or both (Google, 2013). The e-mail and online interface of Groups can be embedded in a Google-hosted course website to facilitate collaboration in the classroom. In particular, the real-time editing in Google Groups can make it easier for students to work collaboratively to share research findings and work together on group projects regardless of their physical location.

\section{MISCONCEPTIONS ABOUT THE CLOUD-BASED GAFE}

The aspect of GAFE that raises the greatest concern among students, faculty, school administrators, and every user is the protection of confidential data, such as personally identifiable information. The protection of users' cross-border and personal data poses a challenge to many providers in terms of data security (Wolf, 2012). The general concern about data protection, as a result of the growth of cloud computing, underscores tensions among cloud service providers, academic communities, data protection regulators, and businesses (Desai, 2013). As the GAFE platform is a cloudbased service, Google takes stringent measures to preserve data integrity and user privacy to maintain 
user confidence. Critical security features exist that are specifically designed to keep user data safe and secure under the user's control (Google, 2013). According to Google (2013), its data center network provides extraordinary security and guarantees protection of data. Additionally, the data centers are designed to ensure automatic data backup on Google servers to ensure approximately $100 \%$ uptime in case of unexpected computer crashes. According to Google, its robust disaster recovery plan is designed to combat exploitable vulnerabilities that may exist within the network. Moreover, evidence shows that Google Apps and the associated data centers are SSAE 16/ISAE 3402 Type II SOC compliant with ISO 27001 certification (Google, 2013), which safeguards user data protection compliance.

Further, Google has integrated security features for e-mail, including spam blocking, virus scanning, and SSL encryption (Google, 2013; Herrick, 2009). Google makes the point that user data are safe from exploitation by other organizations despite the fact that the data sit on their servers. Within both the education and corporate realms, protection of sensitive data in the cloud should be a priority (Stein et al., 2013). Hodgkinson (2012) confirmed that cloud service, such as Google Apps, can live up to expectations by offering better, faster, less expensive, and less risky technology services. According to Google (2013), when in transit, the extra layer of security with two-step verification and the automatic browser sessions with SSLbased encryption offered by Google Apps help protect privacy of users (e.g., students and teachers). Kaganer et al. (2013) noted that the road to a sustainable mobile learning environment will face many challenges.

While security concerns in cloud computing are on the rise, the benefit-to-risk ratio could determine one's decision to adopt the technology (Appling, 2012; Paul, Talreja, Sahu, \& Singh, 2012; Singh, Kharbanda, \& Kaur, 2012). The research community continues to investigate cloud security concerns with the aim of finding better solutions to boost user confidence. Even governments find themselves in the dilemma of how to benefit from cloud technology while maintaining authority over digital data, which is different from physical control (Irion, 2012). However, others have proposed solutions to address these cloud issues. For example,
SeungHwan, Gelogo, and Park (2012) stated that digital IDs can be used to minimize unauthorized access and address nonrepudiation issues. While misconceptions about the cloud-based GAFE may have an adverse impact on its adoption, research should focus on key determinants of GAFE acceptance and offer practical implications for administrators and students to improve productivity and performance.

\section{TARGET POPULATION, SAMPLING, AND METHODOLOGY}

The target population for this study was a section of UG computer science students who were at least 18 years of age. An online survey was used for data collection and administered via a SurveyMonkey online portal. SurveyMonkey (2014) is a provider of web-based survey services around the world. Additionally, a five-point Likert scale ranging from five ("strongly agree") to one ("strongly disagree") was used to measure each item on the questionnaire.

The minimum required sample size for this study was computed using $\mathrm{G}^{*}$ Power, with a power of 0.95 , an effect size of 0.80 , a medium scale, and a level of significance of 0.05 . Considering multiple linear regressions, the computed minimum sample size was 72 based on a $95 \%$ confidence interval.

The researcher used a quantitative research design guided by the following research questions:

1. Is there a statistically significant relationship between student performance and the use of Google Apps for Education?

2. Is there a statistically significant relationship between student performance and intention to use Google Apps for Education?

\section{DATA ANALYSIS AND RESULTS}

The quantitative data were analyzed using SPSS 22 and multiple regression techniques. The variance inflation factor (VIF) and tolerance methods were used to evaluate the degree of multicollinearity in the regression analysis. Descriptive analysis was conducted using a set of variables relevant to the research objectives to describe the data and to make inferences to the research population. 


\section{DEMOGRAPHIC AND DESCRIPTIVE STATISTICS OVERVIEW}

Students' ages, genders, majors, and use of GAFE tools were analyzed based on the data collected. Males represented $64.8 \%$ of the respondents, while females represented $22.5 \%$. Of those surveyed, $71 \%$ were $18-20$ years old, $22.6 \%$ were within $21-22$ years old, while $6.5 \%$ were within 23 24 years old. More than half $(56.5 \%)$ of the students were information technology majors, while $43.5 \%$ were computer science majors.

As shown in Table 1 , nearly $29 \%(n=56)$ of participants used Gmail; this constituted the largest percentage of GAFE users, followed by Google Sites users $(23 \%, n=44)$. Of those users, about $20 \%$ said they often use Google Chrome. In addition, the data revealed that nearly $88 \%$ of those surveyed used at least one Google App. It was not surprising to find that only $1 \%$ of participants used Chromebook, as this is a new and emerging Google product.

\section{Table 1}

\begin{tabular}{|c|c|c|}
\hline \multicolumn{2}{|c|}{ Students' Google Apps/Device Usage } & \\
\hline & $\mathrm{n}$ & $\%$ \\
\hline Gmail & 56 & 28.4 \\
\hline Google Sites & 44 & 22.3 \\
\hline Chrome Browser & 38 & 19.3 \\
\hline Google Drive & 29 & 14.7 \\
\hline Google Group & 12 & 6.1 \\
\hline Google Calendar & 9 & 4.6 \\
\hline Google Talk & 7 & 3.6 \\
\hline Chromebook & 2 & 1.0 \\
\hline Total & 197 & 100.0 \\
\hline
\end{tabular}

The challenges facing the students when using the course website were analyzed. As noted in Table 2, about $24 \%$ of participants encountered no obstacles in using the course website; unfortunately, a larger percentage of respondents (nearly $57 \%$ ) reported connectivity problems. This is not surprising, as bandwidth usage on campus is sometimes an issue. Approximately 10\% reported limited or no access to computers. However, a fewer number of participants reported lack of basic computer skills, lack of computer software skills, lack of Internet security or trust, as well as other limitations, such as financial constraints or family issues (see Table 2).
Table 2

\begin{tabular}{|c|c|c|} 
Student Participants' Obstacles in Using the Course Website \\
\hline Obstacles in Using the Course Website & \multicolumn{2}{|c|}{ Responses } \\
\hline & $\mathrm{n}$ & $\%$ \\
\hline Limited or no Internet connectivity & 41 & 56.9 \\
\hline No obstacles & 17 & 23.6 \\
\hline Limited or no access to a computer & 7 & 9.7 \\
\hline Lack of internet security or trust & 3 & 4.2 \\
\hline Lack of basic computer skills & 2 & 2.8 \\
\hline Lack of computer software skills & 1 & 1.4 \\
\hline Other (e.g., financial constraints, family issues, etc.) & 1 & 1.4 \\
\hline Total & $\mathbf{7 2}$ & $\mathbf{1 0 0 . 0}$ \\
\hline
\end{tabular}

\section{SUMMARY OF ANALYSIS AND RESULTS}

The mean and standard deviation of each variable and the summary statistics of the data set were analyzed for variability and normality. The distributions of the data set were examined for normality with skewness and kurtosis statistics. The values of skewness were within +2 , while those of kurtosis were less than 7. These values are considered to approximate normality (Field, 2013). Hence, the statistical values obtained from the study's results were within acceptable ranges for normality. For illustrative purposes, a histogram of performance showing normal distribution is presented in Figure 1. These indications are attributed to a fairly normal distribution

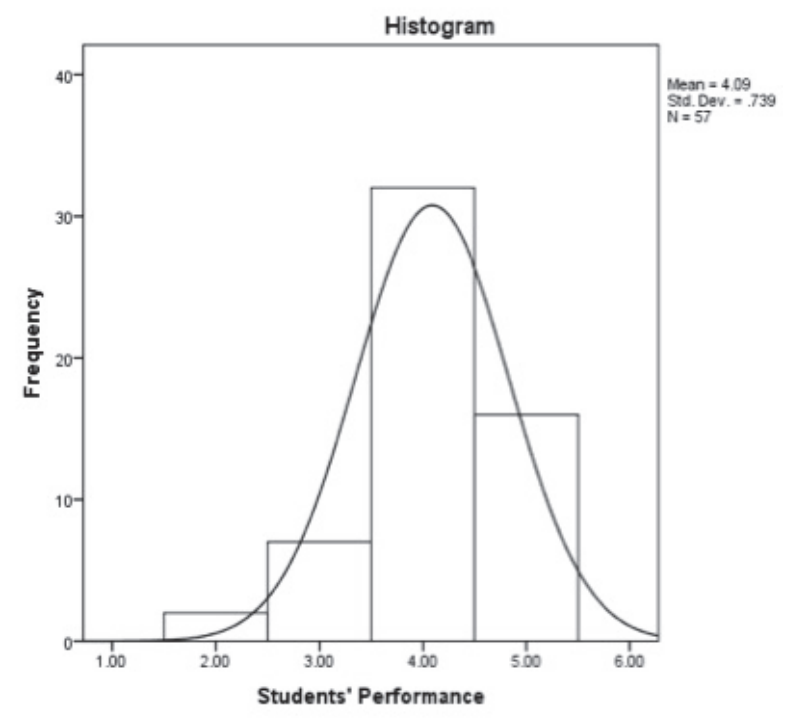

Figure 1. Histogram of performance with superimposed normal distribution curve.

The degree of multicollinearity indicated that the predictor variables were not highly correlated. 
The highest correlation occurred between confidence gained in using the website and flexibility and easy access to course materials, which is significant at a .05 level $(\mathrm{r}=.601, \mathrm{p}=.000)$. Of all of the predictors, knowledge acquired as a result of using the course website correlated best with performance (the outcome variable; $r=.550, p<.000$ ). Therefore, there is a strong likelihood that knowledge gained best predicts performance.

Furthermore, the value of R 2 is .310, which implies that the predictors (i.e., learning impact, confidence, and ease of access to course materials) account for $31.0 \%$ of the variation in performance. In addition, the $\mathrm{F}$ ratio was 5.85 , which is significant at $p<.001$. As the model fit tests the model's ability to predict the dependent variable (Field, 2013), this is an indication that the regression model overall predicts student performance to some degree.

The analysis of variance (ANOVA) describes how the model significantly results in a good degree of prediction of the outcome variable (i.e., performance). For research question one, the ANOVA for the model was statistically significant $(F[1,56]$ $=5.85, \mathrm{p}<.001, \mathrm{R} 2=.310)$. There was a significant, positive relationship between student performance and knowledge gained from using the website $(\beta=$ $.567, \mathrm{t}=3.734, \mathrm{p}<.001)$. The value of .310 for the ANOVA result means that $31 \%$ of the variance in performance can be explained by acquired knowledge from using the GAFE-based course website.

Additionally, the VIF indicates that predictors have strong linear relationships with each other, as the VIF values are far less than 10, while the tolerance statistic maintains values far greater than 0.1 (Field, 2013). Furthermore, the linear fit coefficients and confidence intervals for each predictor variable and the intercept were combined to form the regression equation of the linear fit to predict performance from the predictors.

Using knowledge gained as a predictor, the scatter plot in Figure 2 shows the line of best fit for the data set as indicated in the following equation: $\mathrm{y}=1.64+0.58 * \mathrm{x}$, where $\mathrm{y}$ represents performance and $\mathrm{x}$ represents knowledge gained using the course website.

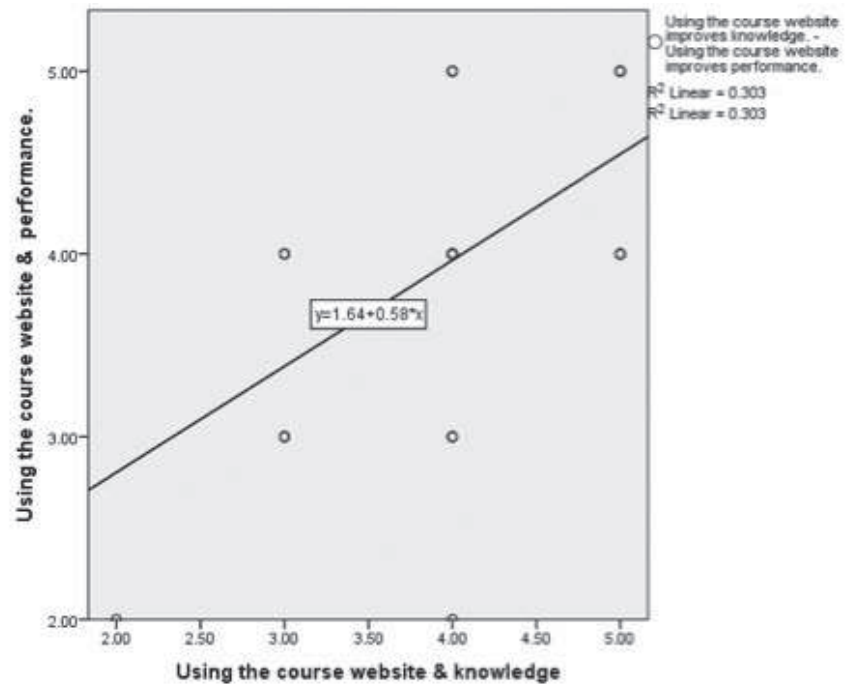

Figure 2. Scatter plot and model of the data set.

\section{DISCUSSION OF THE FINDINGS}

Analysis of the results revealed a significant, positive relationship between student performance and knowledge gained from using the website. In addition, $31 \%$ of the variance in performance was explained by acquired knowledge from using the GAFE-based course website. There were significant, positive relationships between performance and satisfaction and between performance and students' intention to use the course websites and GAFE Apps in future classes. Contrary to expectations, there were no statistically significant relationships between student performance and flexibility (or confidence) as a result of using the apps. As GAFE are among the few widely used tools for collaborations at academic institutions, further research is needed to improve the generalizability of the findings.

\section{LIMITATIONS AND RECOMMENDATIONS FOR FUTURE RESEARCH}

While rigorous measures were applied in the study, certain limitations compromised the validity of the research, which could be addressed in future studies. The first limitation pertains to generalizability of the findings, as the study was conducted in an environment that has a very low penetration rate for Google Apps. The findings may not be applicable to populations that are more technologically advanced or that have high Google Apps adoption rates. Future research should be directed towards wider inclusion criteria to account for a 
more acceptable generalization of the research outcomes. Additionally, data collection was limited to one department at one university. Future studies could focus on extending the scope of the research by collecting data from other departments and/or universities. In particular, given that use behavior differs across age, gender, and experience (Venkatesh et al., 2012), future studies should engage a random sampling approach to include students and academic staff at different levels.

Furthermore, future research could focus on technology adoption theories to provide greater insights into the changing trend of technology use, or diffusion of the innovations, within the academic establishments. For example, to understand how GAFE can potentially permeate society, diffusion of innovations theory can be used to explain how, why, and at what rate GAFE innovations spread through cultures. Furthermore, as perceived ease of use and perceived usefulness are generally known to be key determinants of intention to using a computing system, future research is recommended to investigate these variables as they relate to GAFE adoption.

Another potential area for further study relates to the need for gaining a deeper understanding of the impact of GAFE from the perspective of a unified theory of acceptance. If future research could use the unified theory of acceptance and use of technology to evaluate GAFE adoption, it may help explain usage behavior in more detail. An important consideration could be why some variables were statistically insignificant. Finally, this study did not consider the role of risk and trust in predicting intention and use behavior. As a consequence, additional research should be directed toward investigating perceptions of risk and trust.

\section{CONCLUSION}

The study provided an understanding of GAFE adoption on campus and the potential impact on student performance. In other words, the objective for the research study was to explore the applicability of Google Apps in the classroom. The study has practical implications for researchers and educators. This study was one of the first to investigate adoption and efficient use of GAFE in teaching and learning, while providing an understanding of how GAFE use predicts student performance and intention to adopt the innovation. Based on the statisti- cal analysis, GAFE use and adoption can help improve teaching and learning. While the study had inherent limitations, it could serve as a benchmark for further studies. Furthermore, the findings of this research advanced the practical understanding of GAFE adoption, while further underlining the vital role GAFE plays in facilitating teaching and learning. As an effective cloud-computing solution, which works for users anywhere and at any time, collaborations among students and academic staff could significantly improve teaching and learning if adopted and used effectively.

\section{References}

Allen, E., \& Seaman, J. (2014). Grade change: Tracking online education in the United States. Retrieved from http://www. onlinelearningsurvey.com/reports/gradechange.pdf

Appling, J. G. (2012). We're going Google!: Making the most of marketing. Proceedings from the ACM SIGUCCS Conference, 223-226. doi:10.1145/2382456.2382511

Barlow, K., \& Lane, J. (2007). Like technology from an advanced alien culture: Google Apps for Education at ASU. Proceedings from the ACM SIGUCCS Conference, 8-10. doi:10.1145/1294046.1294049

Cheung, R., \& Vogel, D. (2013). Predicting user acceptance of collaborative technologies: An extension of the technology acceptance model for e-learning. Computers \& Education, 63, 160-175. doi:10.1016/j.compedu.2012.12.003

Cooper, C. (2014). Microsoft's OneDrive to take on Google Drive and Dropbox. Retrieved from http://news.cnet.com

Coursera. (2014). Take the world's best courses, online, for free. Retrieved from https://www.coursera.org/

Desai, D. (2013). Beyond location: Data security in the 21st century. Communications of the ACM, 56(1), 34-36. doi:10.1145/2398356.2398368

Despotović-Zrakić, M., Marković, A., Bogdanović, Z., Barać, D., \& Krčo, S. (2012). Providing adaptivity in Moodle LMS courses. Journal of Educational Technology \& Society, 15(1), 326-338

DiScipio, T. (2013). Essential collaboration, Web 2.0 and social learning platforms for 21st-century education. District Administration, 49(1), 56-57.

Enis, M. (2013). Mobile evolution. Library Journal, 138(2), 34-36.

Field, A. (2013). Discovering statistics using IBM SPSS statistics (4th ed.). Los Angeles, CA: Sage. 
Fu, H., Chu, S., \& Kang, W. (2013). Affordances and constraints of a wiki for primary-school students' group projects. Journal of Educational Technology \& Society, 16(4), 85-96.

Gewerc, A., Montero, L., \& Lama, M. (2014). Collaboration and social networking in higher education. Comunicar, 21(42), 55-62. doi:10.3916/C42-2014-05

Google. (2013). Discover a better way of learning: Free web-based email, calendar \& documents for collaborative study anytime, anywhere. Retrieved from http://www.google.com/enterprise/ apps/education

Google plans Google play for education. (2013). Electronic Education Report, 20(12), 1-4.

Herrick, D. R. (2009). Google this!: Using Google Apps for collaboration and productivity. ACM SIGUCCS, 55-64. doi:10.1145/1629501.1629513

Hodge, K., \& Harman, L. (2013). Technology from Gutenberg to Google and the plastic brain. Curriculum \& Teaching Dialogue, 15(1/2), 111-114.

Hodgkinson, S. (2012). Seeding cloud catalysts. Government News, 32(5), 20-21.

Huffman, S. (2013). Benefits and pitfalls: Simple guidelines for the use of social networking tools in K-12 education. Education, 134(2), 154-160.

Irion, K. (2012). Government cloud computing and national data sovereignty. Policy \& Internet, 4, 40-71. doi:10.1002/poi3.10

Israel, M., Marino, M. T., Basham, J. D., \& Spivak, W. (2013). Fifth graders as app designers: How diverse learners conceptualize educational apps. Journal of Research on Technology in Education, 46(1), 53-80.

Junco, R. R., Heiberger, G. G., \& Loken, E. E. (2011). The effect of Twitter on college student engagement and grades. Journal of Computer Assisted Learning, 27(2), 119-132. doi:10.1111/ j.1365-2729.2010.00387.x

Kaganer, E., Giordano, G. A., Brion, S., \& Tortoriello, M. (2013). Media tablets for mobile learning. Communications of the ACM, 56(11), 68-75. doi:10.1145/2500494

Klein, R., Orelup, R. M., \& Smith, M. (2012). Google Apps for Education: Valparaiso University's migration experience. Proceedings from the ACM SIGUCCS Conference, 203-208. doi:10.1145/2382456.2382506

Lee, J.H., \& Song, C.H. (2013). Effects of trust and perceived risk on user acceptance of a new technology service. Social Behavior \& Personality: An International Journal, 41(4), 587-597. doi:10.2224/sbp.2013.41.4.587
Lin, S., Zimmer, J.C., \& Lee, V. (2013). Podcasting acceptance on campus: The differing perspectives of teachers and students. Computers \& Education, 68, 416-428. doi:10.1016 /j.compedu.2013.06.003

Nistor, N., Göğüş, A., \& Lerche, T. (2013). Educational technology acceptance across national and professional cultures: A European study. Educational Technology Research \& Development, 61(4), 733-749. doi:10.1007/s11423-013-9292-7

Nistor, N., Lerche, T., Weinberger, A., Ceobanu, C., \& Heymann, O. (2014). Towards the integration of culture into the unified theory of acceptance and use of technology. British Journal of Educational Technology, 45(1), 36-55. doi:10.1111/j.14678535.2012.01383.x

Norman, H., Din, R., Nordin, N., \& Ryberg, T. (2014). A review on the use and perceived effects of mobile blogs on learning in higher educational settings. Asian Social Science, 10(1), 209-222. doi:10.5539/ass.v10n1p209

Paul, R., Talreja, M., Sahu, A., \& Singh, K. (2012). Security issues in cloud computing. International Journal on Computer Science \& Engineering, 4(11), 1863-1867.

Pritchett, C., Wohleb, E., \& Pritchett, C. (2013). Educators' perceived importance of Web 2.0 technology applications. Techtrends: Linking Research \& Practice to Improve Learning, 57(2), 33-38. doi:10.1007/s11528-013-0643-3

Ravi Shankar, P. P. (2012). Coursera: Free online learning for the world. Australasian Medical Journal, 5(11), 600-601.

Robertson, C. (2013). Using a cloud-based computing environment to support teacher training on common core implementation. Techtrends: Linking Research \& Practice To Improve Learning, 57(6), 57-60. doi:10.1007/s11528-013-0702-9

Rock, M. L., Schoenfeld, N., Zigmond, N., Gable, R. A., Gregg, M., Ploessl, D. M., \& Salter, A. (2013). Can you Skype me now? Developing teachers' classroom management practices through virtual coaching. Beyond Behavior, 22(3), 15-23.

Scheuer, M. (2013). Book apps as a new interactive learning experience: Evaluating and reviewing this new media. CSLA Journal, 17-20.

SeungHwan, J., Gelogo, Y.E., \& Park, B. (2012). Next generation cloud computing issues and solutions. International Journal of Control \& Automation, 5(1), 63-70.

Singh, K., Kharbanda, I., \& Kaur, N. (2012). Security issues occur in cloud computing and their solutions. International Journal on Computer Science \& Engineering, 4(5), 945-949. 
Sloan Consortium (2013). Changing Course: Ten Years of Tracking Online Education in the United States. Retrieved from http:// www.onlinelearningsurvey.com/reports/changingcourse.pdf

Stein, S., Ware, J., Laboy, J., \& Schaffer, H. (2013). Improving $\mathrm{K}-12$ pedagogy via a cloud designed for education. International Journal of Information Management, 33(1), 235-241.

Suk Hwang, Y., \& Vrongistinos, K. (2012). Using Blackboard and Skype for mentoring beginning teachers. American Journal of Distance Education, 26(3), 172-179. doi:10.1080/08923647.2 012.697019

SurveyMonkey. (2014). Create surveys. Get answers. Retrieved from https://www.surveymonkey.com

Sviridova, T., Sviridova, L., \& Tymoshenko, B. (2011). Google Apps as solution of communication issues in educational process. Proceedings from IEEE 7th International Conference, 183-184.

Teo, T., \& Noyes, J. (2014). Explaining the intention to use technology among pre-service teachers: A multi-group analysis of the unified theory of acceptance and use of technology. Interactive Learning Environments, 22(1), 51-66. doi:10.1080/ 10494820.2011.641674

Ucol-Ganiron, T., Jr. (2013). Web-enhanced project management course. International Journal of U- \& E-Service, Science \& Technology, 6(1), 49-60.

Veletsianos, G. G. (2012). Higher education scholars' participation and practices on Twitter. Journal of Computer Assisted Learning, 28(4), 336-349. doi:10.1111/j.13652729.2011.00449.x

Venkatesh, V. L., Thong, J. Y., \& Xu, X. (2012). Consumer acceptance and use of information technology: Extending the unified theory of acceptance and use of technology. MIS Quarterly, 36(1), 157-178.

Vural, Ö. (2013). The impact of a question-embedded video-based learning tool on e-learning. Educational Sciences: Theory \& Practice, 13(2), 1315-1323.

Wang, Y., \& Jin, B. (2010). The application of SaaS model in network education-Take Google Apps for example. Education Technology and Computer, 4, 191-194.

Wolf, C. (2012). Privacy and data security in the cloud: What are the issues? IP Litigator, 18(6), 19-28.

Zolotukhin, S. (2013). The application of the Web 2.0 technology as a tool of developing informal media education. Vestnik IKBFU, (11), 39-45.

\section{Author Biography}

Lawrence Awuah earned his $\mathrm{PhD}$ degree in Information Technology with specialization in Information Assurance, MS degree in electrical \& computer engineering, and BS degree in electrical and electronics engineering. He has several years of experience working in the industry in the fields of electrical engineering, networking, and information security. Lawrence is currently a Professor of Information Technology Department at Colorado State University-Global Campus and an Adjunct Faculty of College of Science, Engineering and Technology at Grand Canyon University. He serves on the Executive Advisory Board, School African Foundation. Further, he is an educational technology consultant who helps educators integrate technology and curriculum in their programs. His primary research interests include high performance computing (HPC), software defined networking (SDN), mobile computing, networking, technology acceptance, diffusion of innovations, and application of technology in education. 